\title{
M 82's stellar bar
}

\author{
A. Greve ${ }^{1}$, K. A. Wills ${ }^{2}$, N. Neininger ${ }^{3}$, and A. Pedlar ${ }^{4,5}$ \\ 1 Institut de Radio Astronomie Millimétrique, 300 rue de la Piscine, 38406 St. Martin d'Hères, France \\ 2 Department of Physics and Astronomy, University of Sheffield, Hounsfield Road, Sheffield S3 7RH, UK \\ 3 Astronomisches Institut der Universität Bonn, Auf dem Hügel 71, 53121 Bonn, Germany \\ 4 University of Manchester, Jodrell Bank Observatory, Macclesfield, Cheshire SK11 9DL, UK \\ ${ }^{5}$ Onsala Space Observatory, 43992 Onsala, Sweden
}

Received 28 September 2001 / Accepted 26 November 2001

\begin{abstract}
The fueling of the starburst in M 82 may be related to a stellar bar which pushes gas towards the center where it forms stars. The observation by McKeith et al. (1993) of the near-IR Ca II photospheric absorption line allows a direct velocity measurement of the stars in M 82, and provides by this a confirmation of the predicted $x_{1}$ and $x_{2}$-orbits of the bar in M 82 . From this and other observations we find that the mass of the $x_{2}$-orbit stars is $\sim 15 \%$ of the mass of the bar, and that the mass of the bar of $2 \times 10^{9} M_{\odot}$ is $20-40 \%$ of M 82 's mass. This mass concentration of $\sim 1 \mathrm{kpc}$ extent at the center of M 82 underlines the dynamic importance of the bar.
\end{abstract}

Key words. galaxies: individual: M 82 - galaxies: structure - galaxies: stellar content

\section{Introduction}

The starburst in M 82, of approximately 10-50 Myr age, can possibly be explained by the presence of a stellar bar which pushes gas towards the center of the galaxy where it accumulates, is compressed, shocks, and forms stars. This stellar bar may have formed during the encounter of M 81 and M 82, several 100 Myr ago. Tidal arms visible in $21 \mathrm{~cm}-\mathrm{H}$ I still connect M 81, M 82, and NGC 3077.

In a bar, the stars move on elliptical orbits along the major axis of the bar, the $x_{1}$-orbits, and at the center of the galaxy on perpendicular orbits along the minor axis of the bar, the $x_{2}$-orbits. The consequence for the gas distribution is motion of gas from the far ends of the bar towards the center, shocks at the intersection of the $x_{1}$ and $x_{2}$-orbits, accumulation of gas near and inside the $x_{2}$-orbits, and the formation of dust lanes along the leading $x_{1}$-orbits (Contopoulos \& Mertzanides 1977; Binney et al. 1991; Athanassoula 1992). Morphological evidence for the bar in M 82 comes from the box-shaped distribution of stellar light, in particular measured in the $2.2 \mu \mathrm{m} \mathrm{K}$-band which emphasizes late-type stars (Telesco et al. 1991; Larkin et al. 1994), and the indication of dust lanes from IR extinction measurements of $x_{1}$-orbit stars (Larkin et al. 1994). Kinematic evidence of the $x_{1}$-orbits comes from position-velocity $(p-v)$ measurements of neutral and ionized gas (CO, H I, $\mathrm{H}_{\alpha}$, etc.; Shen \& Lo 1995; Seaquist et al. 1996; Neininger et al. 1998; Wills et al. 2000; McKeith et al. 1993). Kinematic evidence of the $x_{2}$-orbits can be seen in the $\mathrm{CO}$ and $\mathrm{HI}$ data, however it is

Send offprint requests to: A. Greve, e-mail: greve@iram.fr most clearly present in the $p-v$ diagrams obtained from the $12.8 \mu \mathrm{m}$ [NeII] emission line (Achtermann \& Lacy 1995). In fact the ionized gas traced out by the [NeII] emission appears to be largely confined to the $x_{2}$-orbits only. There are no direct velocity measurements of the stars of the bar which may be different from the velocity of the gas, in particular in regions of shocks, dust lanes, and bubbles. Direct information on the stars is important since they contain the dominant mass of the bar and define the gravitational potential in which the material moves.

Here we show that the earlier single long-slit spectroscopic observation by McKeith et al. (1993) of the near-IR Ca II photospheric absorption lines (at 8662, 8542, $8498 \AA$ ), which are strong in late type stars (Jaschek \& Jaschek 1995), provides direct kinematic evidence of the stellar $x_{1}$-orbits and $x_{2}$-orbits of M 82 . The observations of near-IR emission lines of $\mathrm{H}$ II regions, like [SIII] at $9069 \AA$ and Paschen-10 $[\mathrm{Pa}(10)]$ at $9014 \AA$, however do not immediately reveal the $x_{2}$-orbits, at least at the selected slit position discussed here. Evidently, the stellar absorption lines can only be observed where the stellar continuum is strong, which is along the major axis of the bar and along the more extended major axis of the galactic disk. We derive from the $p-v$ diagrams the extent and the mass of the bar.

\section{Observation}

The observation used in this publication was described earlier (McKeith et al. 1993) and was made to investigate the rotation of gas and stars in M 82, which were previously claimed to be different (Saito et al. 1984). The single 


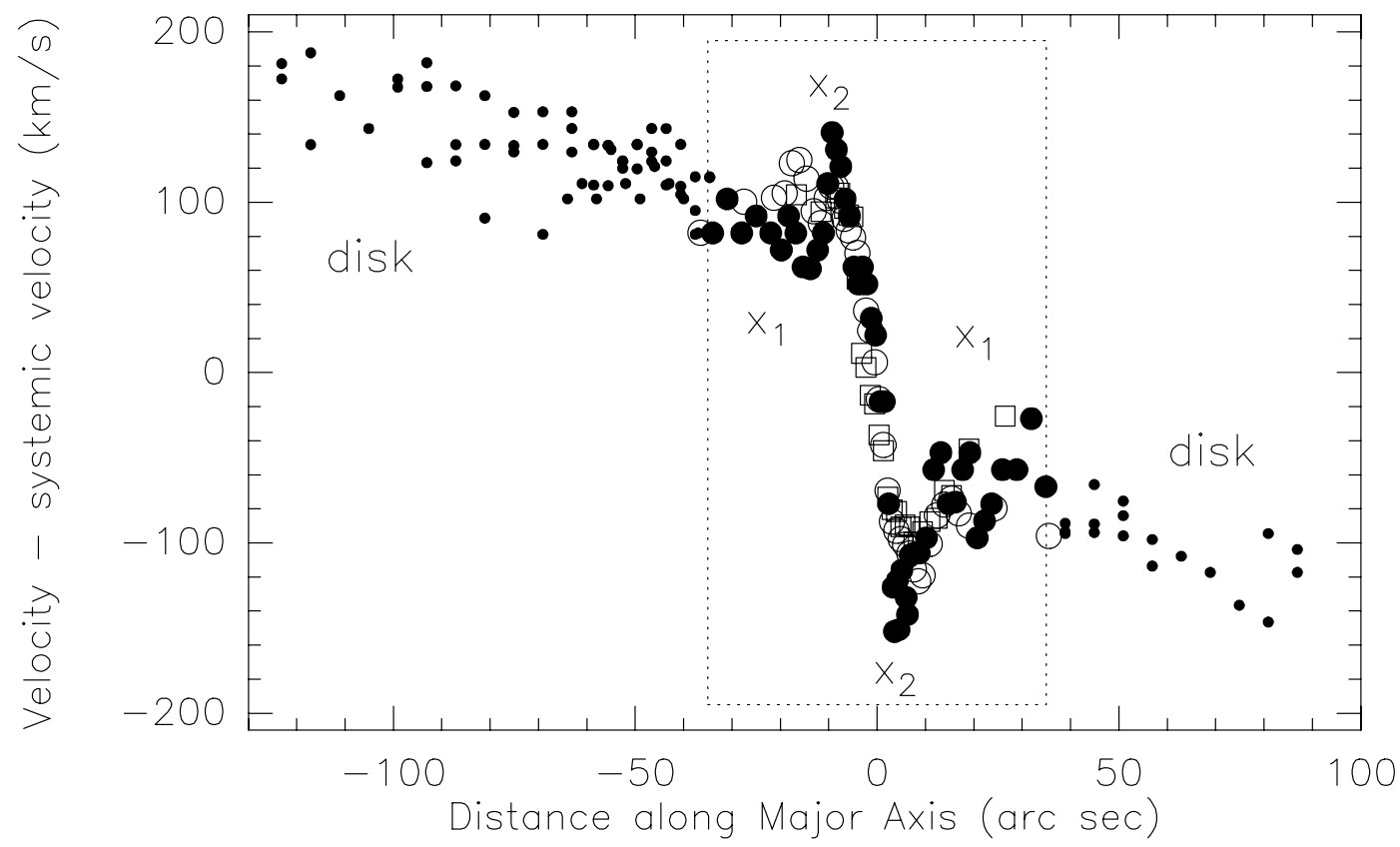

Fig. 1. Major axis $\left(\mathrm{PA}=65^{\circ}\right) p-v$ diagram of the stellar bar $\left(\theta \lesssim 30^{\prime \prime}\right.$, i.e. $r \lesssim 500$ pc: heavy symbols in the outlined region $)$ and of the disk component. The extent of $\pm 100^{\prime \prime}$ corresponds to \pm 1700 pc. Near-IR Ca II stellar absorption lines: heavy and small dots; near-IR ionized gas emission lines [SIII]: open circles, $\mathrm{Pa}(10)$ : open squares. The systemic velocity of $200 \mathrm{~km} \mathrm{~s}{ }^{-1}$ is subtracted. ENE is to the left, WSW to the right. $1^{\prime \prime}$ is equivalent to $17.5 \mathrm{pc}$.

long-slit spectroscopic observation, made with the $4.2 \mathrm{~m}$ WHT (La Palma, Spain) along the major axis of M 82 $\left(\mathrm{PA}=65^{\circ}\right)$, covered the wavelengths from $[\mathrm{OII}] 3729 \AA$ to $[\mathrm{SIII}] 9069 \AA$ with a velocity resolution of $\sim 30 \mathrm{~km} \mathrm{~s}^{-1}$. The seeing was good and of the order of $0.7^{\prime \prime}$. The velocity information of the stellar Ca II photospheric absorption line at $8542 \AA$ (strongest triplet line) and of the ionized gas emission lines $[\mathrm{SIII}]$ and $\mathrm{Pa}(10)$, taken from the observation by McKeith et al. (1993), is summarized in Table 1. The extreme velocities (indicated by the dots in Table 1$)$, which reveal the $x_{2}$-orbit stars, are confined to narrow regions $\sim 2.5^{\prime \prime}(\sim 45 \mathrm{pc})$ in extent, at approximately $5^{\prime \prime}$ distance on either side of the dynamical center. These extreme velocities are only detectable under good (sub-arcsec) seeing conditions.

The investigation by McKeith et al. (1993) revealed that at the same location in M82 the rotation of the gas and stars is globally the same, and that the apparent rotation curve becomes steeper at longer wavelengths and in smaller regions at the center. McKeith et al. explained these features as an effect of extinction which hides at blue wavelengths the rapidly rotating core. They also found that the velocity measured in the direction of the center from near-IR ionized gas emission lines and near$I R$ stellar absorption lines is close to the velocity of the $12.8 \mu \mathrm{m}[\mathrm{NeII}]$ emission line (Beck et al. 1978), which is unaffected by extinction and probes the center of M 82 . They concluded therefore that - despite an average visual extinction of $A_{\mathrm{V}} \approx 3-8$ mag towards the center region ${ }^{1}$ (Telesco et al. 1991; Larkin et al. 1994) - the near-IR lines, and in particular the near-IR Ca II stellar absorption lines, probe also the center of the galaxy or regions very close to it. Today we are able to interpret this earlier observation of the near-IR CaII line in the light of the proposed stellar bar.

The major axis $p-v$ diagram of M 82, based on the observation by McKeith et al. (1993; including Table 1), of the region extending to $\sim \pm 100^{\prime \prime}( \pm 1700 \mathrm{pc})$ on either side of the galactic center and containing the bar is shown in Fig. 1. This figure is mainly constructed from the stellar CaII line and thus shows in essence the motion of stars. The velocities display the rotation of M 82 .

The equivalent widths of the CaII triplet lines (see McKeith et al. 1993, their Fig. 1) are $W(8498) \approx 1.3 \AA$, $W(8542) \approx 3.4 \AA$, and $W(8662) \approx 2.5 \AA$, indicating primarily G-K III-V type stars (Jaschek \& Jaschek 1995) in agreement with the fact that the bar consists of late-type stars which were spatially reshuffled in the encounter with M 81. Similar stellar types are derived by Förster Schreiber et al. (2001).

Distances along M 82's major axis are originally measured in angular scale $(\theta)$. For a distance of $3.6 \mathrm{Mpc}$ (Freedman et al. 1994; Sakai \& Madore 1999), $1^{\prime \prime}$ is equivalent to $17.5 \mathrm{pc}$ linear scale $(r)$.

\footnotetext{
1 Derived from stellar colours, hence relevant to observations of stars but not necessarily of the gas. For a compilation of extinction determinations in M 82 see Götz et al. (1990).
} 


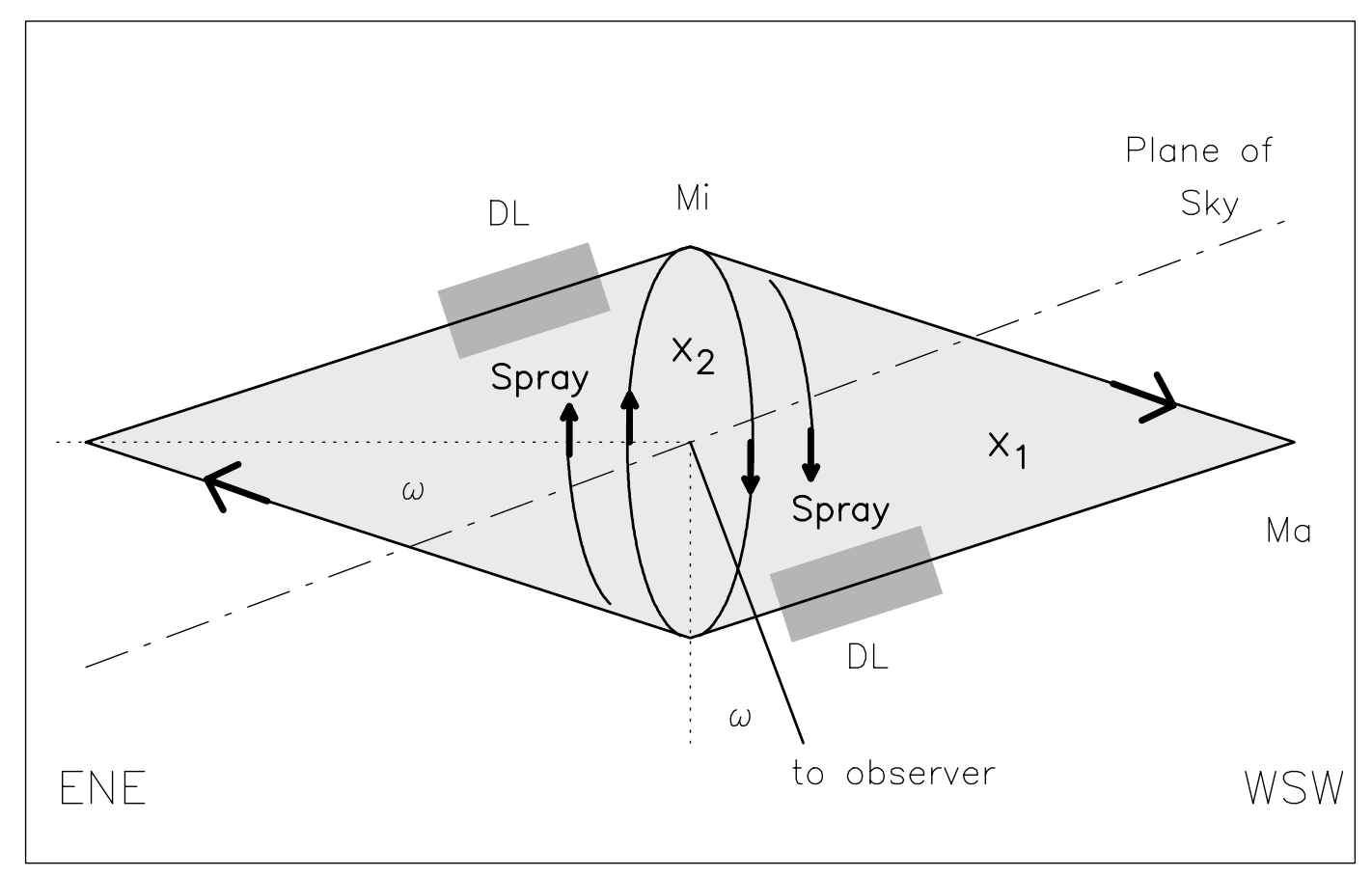

Fig. 2. Schematic explanation of "spraying" in M 82 (not drawn to scale): some gas is pulled off the trailing $x_{1}$-orbits and is pushed towards the leading $x_{1}$-orbits, forming dust lanes (DL). Ma is the major axis, Mi the minor axis of the bar. The region of the $x_{1}$ and $x_{2}$-orbit stars is indicated. The extent of the bar $\left(x_{1}\right.$-orbits $)$ is $\sim 1 \mathrm{kpc}\left(\sim 60^{\prime \prime}\right) ; \omega \approx 22^{\circ}$. The western side of M 82 moves towards the observer, the eastern side away from the observer. M 82 is viewed from the top (North).

\section{The orientation of the bar and "spraying"}

\subsection{Orientation of the bar}

Detailed knowledge of the orientation of the bar, as required to understand the measured $p-v$ diagrams, is not easily available from a single long-slit spectrogram. We use, and summarize, the results obtained by Telesco et al. (1991) and Wills et al. (2000).

The position angle of $\mathrm{M} 82^{\prime}$ 's major axis is $\mathrm{PA} \approx 70^{\circ}$; the western side moves towards the observer, the eastern side away from the observer. In the following we refer to the axis at $\mathrm{PA}=70^{\circ}$ as the "galactic plane". The inclination of M 82 is $i \approx 80^{\circ}$, with the southern side being more distant as for instance suggested by the higher reddening and polarization (Visvanathan \& Sandage 1972; Notni \& Bronkalla 1983; Chesterman \& Pallister 1980), the motion of ionized gas (Götz et al. 1990), and the geometry of the minor axis outflow (McKeith et al. 1995; Shopbell \& Bland-Hawthorn 1998). However, from an observed increase towards the north of free-free absorption due to ionized gas, Wills et al. (2000) discuss the possibility of the opposite orientation. Following Telesco et al. (1991, their Fig. 11), the bar is inclined by $\sim 4^{\circ}$ with respect to M 82's galactic plane, with the western side of the bar lying above the galactic plane. As schematically shown in Fig. 2, the major axis of the bar is turned out of the plane of the sky by $\sim 22^{\circ}(\omega)$, with the eastern side being more distant. We are looking at the major axis of the bar at an angle of $\sim 68^{\circ}$ so that the major axis $p-v$ diagram resembles that shown by Binney et al. (1991, their Fig. 1, left and middle panel; though referring to our Galaxy) and Achtermann \& Lacy (1994, their Fig. 15) for M 82. The orientation of the bar is inverted when using the orientation of M 82 suggested by Wills et al.

The $x_{2}$-orbits are perpendicular to the $x_{1}$-orbits and their major axis is rotated to the line-of-sight by $\omega \approx 22^{\circ}$ (Fig. 2). If the southern side of M 82 is more distant, the $x_{2}$-orbits extend at the eastern side out of the plane of the sky towards the observer. Following Figs. 1 and 3, the $x_{2}$-orbit stars on the north-eastern side move away from the observer (red-shifted), at the south-western side towards the observer (blue-shifted).

The following figures and parameters refer to the geometry and velocities of the bar as seen projected onto the plane of the sky.

\section{2. "Spraying"}

For the interpretation of optical and radio observations of bar galaxies it is important to consider the effect of spraying as predicted from $N$-body calculations (Athanassoula 1992) and used, for instance, to explain the observations of NGC 1530 (Downes et al. 1996). Altough the bars of bar galaxies are massive and several kpc in extent, the $1 \mathrm{kpc}-$ size bar of M 82 (see below) may produce the same effect of spraying. The effect is schematically shown in Fig. 2. When passing the gravitational potential of the $x_{2}$-orbit stars, some gas (and perhaps also some stars) is pulled off the trailing $x_{1}$-orbits and is pushed towards the leading $x_{1}$-orbits. The spraying gas may shock with the gas on 
Table 1. $p-v$ information derived from the near-IR stellar Ca II (8542) absorption line and the [SIII] and $\mathrm{Pa}(10)$ emission lines (from McKeith et al. 1993).

\begin{tabular}{|c|c|c|c|}
\hline \multirow{2}{*}{$\begin{array}{c}\text { Position } \\
\left({ }^{\prime \prime}\right) \\
\end{array}$} & \multirow{2}{*}{$\begin{array}{c}\text { Stellar Velocities } \\
\text { Ca II IR line } \\
\left(\mathrm{km} \mathrm{s}^{-1}\right) \\
\end{array}$} & \multicolumn{2}{|c|}{ Gas Velocities } \\
\hline & & $\begin{array}{c}{[\text { SIII }]} \\
\left(\mathrm{km} \mathrm{s}^{-1}\right)\end{array}$ & $\begin{array}{c}\mathrm{Pa}(10) \\
\left(\mathrm{km} \mathrm{s}^{-1}\right)\end{array}$ \\
\hline \multicolumn{4}{|l|}{$\boldsymbol{x}_{1}$-orbits ${ }^{\text {a) }}$} \\
\hline-34.0 & 82 & & \\
\hline-31.0 & 102 & & \\
\hline-28.0 & 82 & 100 & \\
\hline-25.0 & 92 & & \\
\hline-22.0 & 82 & 102 & \\
\hline-19.8 & 72 & 105 & \\
\hline-18.3 & 92 & 123 & \\
\hline-16.8 & 82 & 125 & 104 \\
\hline-15.3 & 62 & 114 & \\
\hline-13.8 & 61 & 95 & \\
\hline-12.3 & 72 & & \\
\hline-11.1 & 82 & 88 & 94 \\
\hline \multicolumn{4}{|l|}{$\boldsymbol{x}_{2}-$ orbits } \\
\hline-10.2 & 111 & 102 & \\
\hline-9.3 & • 141 & 109 & \\
\hline-8.4 & • 131 & 109 & 102 \\
\hline-7.5 & • 121 & 101 & 105 \\
\hline-6.6 & 102 & 90 & 96 \\
\hline-5.7 & 92 & 83 & 92 \\
\hline-4.8 & 62 & 79 & 91 \\
\hline-3.9 & 52 & 70 & 55 \\
\hline-3.0 & 62 & 53 & 11 \\
\hline-2.1 & 52 & 36 & 3 \\
\hline-1.2 & 32 & 25 & -13 \\
\hline-0.3 & 22 & 6 & -18 \\
\hline 0.6 & -17 & -15 & -36 \\
\hline 1.5 & -17 & -42 & -46 \\
\hline 2.4 & -77 & -69 & -73 \\
\hline 3.3 & $\bullet-126$ & -87 & -80 \\
\hline 3.6 & $\bullet-152$ & & \\
\hline 4.2 & $\bullet-122$ & -93 & -81 \\
\hline 4.5 & $\bullet-151$ & & \\
\hline 5.1 & -116 & -98 & -90 \\
\hline 6.0 & -132 & -101 & -89 \\
\hline 6.3 & -142 & -106 & -90 \\
\hline \multicolumn{4}{|l|}{$\boldsymbol{x}_{1}-$ orbits ${ }^{\mathrm{b})}$} \\
\hline 6.9 & -107 & & \\
\hline 7.8 & -106 & -115 & -100 \\
\hline 8.7 & -106 & -122 & -100 \\
\hline 10.2 & -97 & -118 & \\
\hline 11.7 & -57 & -84 & -87 \\
\hline 13.2 & -47 & -77 & -69 \\
\hline 14.7 & -77 & -74 & \\
\hline 16.2 & -76 & -83 & \\
\hline 17.7 & -57 & & \\
\hline 19.2 & -47 & -90 & -45 \\
\hline 20.7 & -97 & & \\
\hline 22.2 & -87 & & \\
\hline 23.7 & -77 & -80 & \\
\hline 25.9 & -57 & & \\
\hline 28.9 & -57 & & \\
\hline 31.9 & -27 & & \\
\hline 34.9 & -67 & -96 & \\
\hline
\end{tabular}

a) ENE side; b) WSW side of center.

- Extreme $x_{2}$-orbit velocities. the leading $x_{1}$-orbits, and eventually form dust lanes and stars.

\section{The orbits of the bar}

Figure 3 shows an overlay ${ }^{2}$ of the observation of the nearIR stellar Ca II line, the [SIII] and $\mathrm{Pa}(10)$ lines (Table 1), and the $x_{1}$-orbits, cusped orbits, and $x_{2}$-orbits of the bar predicted by Wills et al. (2000). Their model of the bar is based on observations of neutral and ionized gas motions (CO, H I, [NeII]).

\subsection{The $x_{2}$-orbits $\left(\theta \leq 5^{\prime \prime}\right)$}

As evident from Figs. 1 and 3, the near-IR Ca II stellar absorption line clearly traces the $x_{2}$-orbits which extend to $\sim \pm 5^{\prime \prime}(\sim \pm 90 \mathrm{pc})$ from the galactic center where the turn-around velocity is $\sim \pm 150 \mathrm{~km} \mathrm{~s}^{-1}$. The observation of the [SIII] and $\mathrm{Pa}(10)$ gas emission lines does not show the extreme turn-around velocity of the stellar $x_{2}$-orbits. The gas apparently enters the region of the $x_{2}$-orbits at a velocity of $\sim 100 \mathrm{~km} \mathrm{~s}^{-1}$ and follows the rotation of the stellar bar only at the inner $\sim \pm 3^{\prime \prime}(\sim \pm 50 \mathrm{pc})$.

The original kinematic evidence for the $x_{2}$-orbits comes from Achtermann \& Lacy's (1995) $12.8 \mu \mathrm{m}$ [NeII] emission line observation, which covers a radial distance of $\pm 20^{\prime \prime}( \pm 350 \mathrm{pc})$. In this observation the $x_{2}$-orbit gas is seen confined to the central region of $\sim 5^{\prime \prime}$ radius; the gas motion shows the positive and negative velocities of the $x_{2}$-orbits, on both sides of the galactic center (shown by Wills et al., their Fig. 9). Figure 4 shows an overlay of Achtermann \& Lacy's [NeII] line observation and the Ca II line observation of Table 1.

The $21 \mathrm{~cm}-\mathrm{H}$ I observation published by Wills et al. (2000, their Fig. 9) does not clearly show the positive velocities of the $x_{2}$-orbits, and the negative velocities mainly on the southern side of the galactic plane. The $\mathrm{CO}$ observation by Shen \& Lo (1995) (shown by Wills et al., their Fig. 9) shows mainly the negative velocities of the $x_{2}$-orbits on the southern side and in the galactic plane, and marginally the positive velocities in and on the northern side of the galactic plane. ${ }^{12} \mathrm{CO}$ gas on the western side $x_{2}$-orbits is clearly seen in the observation by Weiß et al. (2001). However, they interpret this velocity feature as the eastern part of a super-bubble (see below). ${ }^{12} \mathrm{CO}$ gas on the eastern side $x_{2}$-orbits is marginally seen in Weiß' et al. observation. The recent determination by Weiß et al. of the $\mathrm{H}_{2}$ distribution in M 82 shows a weak central concentration $\left(\sim 2 \times 10^{22} \mathrm{~cm}^{-2}\right.$ column density $)$ of similar extent as the $x_{2}$-orbit region.

The observation by Larkin et al. (1994) shows on the major axis enhanced $\operatorname{Br} \gamma$ emission at a radial distance

\footnotetext{
${ }^{2}$ In the $p-v$ diagram we had to rotate slightly the data of Table 1, or the orbits predicted by Wills. et al., in order to obtain coincidence. This rotation is due to the fact that the slit was at an $\sim 8^{\circ}$ different position angle $\left(65^{\circ}\right)$ than used for the prediction of the orbits $\left(73^{\circ}\right)$. This must be remembered when using the data of Table 1.
} 


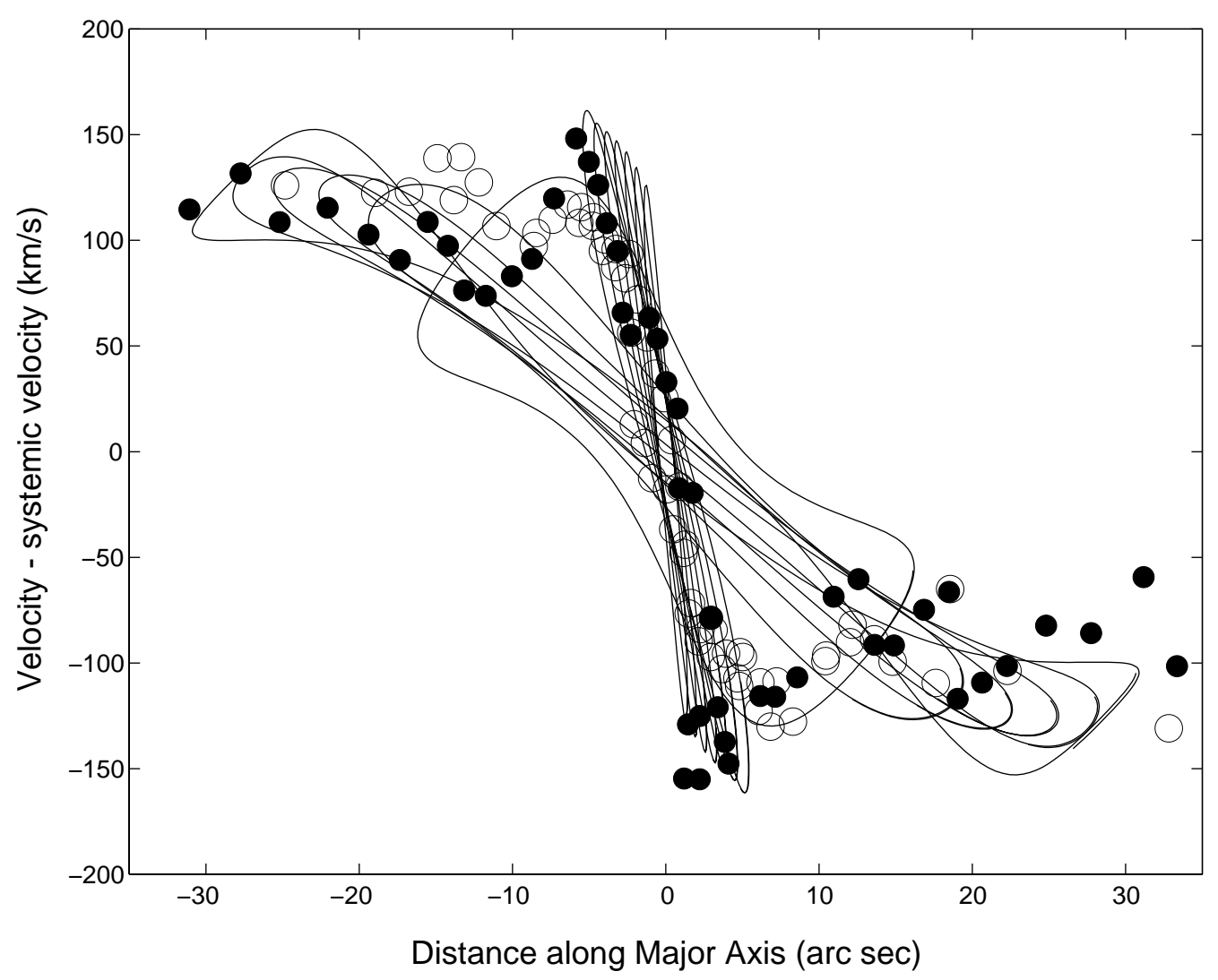

Fig. 3. Major axis $\left(\mathrm{PA}=73^{\circ}\right) p-v$ diagram of the stellar bar region (outlined by the dashed line in Fig. 1). The contour lines show the stellar $x_{2}$-orbits, cusped orbits (peanut-shaped), and $x_{1}$-orbits confined to a region of $\sim \pm 30^{\prime \prime}(\sim \pm 500 \mathrm{pc})$, predicted by Wills et al. (2000). Solid dots: near-IR Ca II stellar absorption line; open circles: near-IR ionized gas emission line of [SIII] and $\mathrm{Pa}(10)$ (data of Table 1). ENE is to the left, WSW to the right. $1^{\prime \prime}$ is equivalent to $17.5 \mathrm{pc}$.

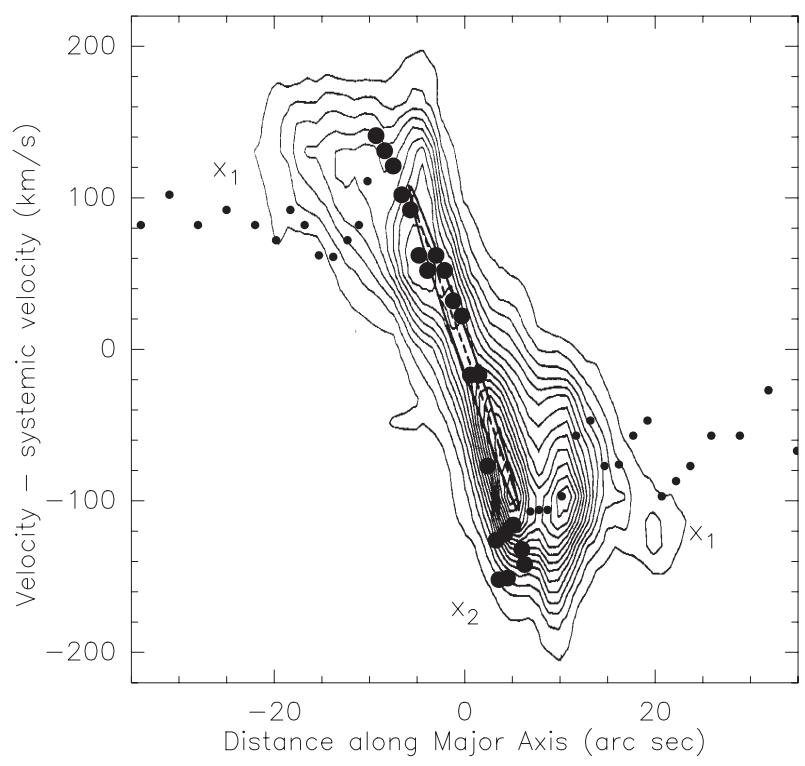

Fig. 4. The contour lines show the velocity of the $12.8 \mu \mathrm{m}$ $[\mathrm{NeII}]$ line measured along M 82's major axis $\left(\mathrm{PA}=70^{\circ}\right)$. The solid line and the dashed line indicates an ionized gas ring, with and without expansion (from Achtermann \& Lacy 1994). Solid dots: near-IR stellar Ca II absorption line (data of Table 1); heavy dots: $x_{2}$-orbits, small dots: $x_{1}$-orbits. of $\sim 4-5^{\prime \prime}$ on either side of the galactic center, i.e. at the edge of the $x_{2}$-region, but mainly on the southern side $\left(0^{\prime \prime}\right.$ to $\left.-3^{\prime \prime}\right)$ of the galactic plane. The bulk velocity of this material at $4-5^{\prime \prime}$ radial distance is $\sim \pm(50-100) \mathrm{km} \mathrm{s}^{-1}$ (Larkin et al., their Figs. 5 and 6 ) while the $x_{2}$-orbit stars at this position have a velocity of $\sim \pm 150 \mathrm{~km} \mathrm{~s}^{-1}$. The discrepancy of the $\operatorname{Br} \gamma$ material velocity, as compared to the stellar velocity discussed here, may be explained, partially, by the low velocity resolution $\left(90 \mathrm{~km} \mathrm{~s}^{-1}\right)$ of Larkin's et al. observation. On the other hand, the $\mathrm{Br} \gamma$ radiating gas is approximately located at the edge of the $x_{2}$-orbits where the gaseous material may "spray", shock, and form stars (Fig. 2), so that some of the gas is at a velocity different from that of the $x_{2}$-orbit stars. The difference of $\sim 50$ $100 \mathrm{~km} \mathrm{~s}^{-1}$ between the stellar velocity and the ionized gas velocity is of the order of the expected shock velocity. This dynamical feature may explain, at least partially, the fact that the ionized gas observed in [SIII] and $\mathrm{Pa}(10)$ does not follow the stellar turn-around velocity at the edge of the $x_{2}$-orbit region but a velocity modified by a superimposed shock (Fig. 3). Telesco et al. (1991) find 10.8, 19.2 and $30 \mu \mathrm{m}$ continuum emission from gas and dust (of a few arcsec extent) concentrated at the far ends of the $x_{2}$-orbits. The $\operatorname{Br} \gamma$ emitting material and the emission features $E_{1}$ and $W_{1}$ measured by Larkin et al. (1994) coincide with the continuum emission observed by Telesco et al. and most likely is the same gas. This gas concentration 
is seen along the tangential line-of-sight of the $x_{2}$-orbits and is assumed to form a ring (Achtermann \& Lacy 1994; Larkin et al. 1994).

The light of the $x_{2}$-orbit stars confined to the radial distance of $\sim \pm 5^{\prime \prime}$ is not separately seen in the $2.2 \mu \mathrm{m}$ major axis profile measured by Telesco et al. (1991, their Fig. 10), but is clearly seen as a central peak in the profile measured by Larkin et al. (1994, their Fig. 2; effective radius $\Theta_{2} \approx 2^{\prime \prime}, R_{2} \approx 35 \mathrm{pc}$, see footnote $\mathrm{c}$ in Table 3 ).

In summary there exists convincing evidence from stellar continuum, stellar absorption line, and gas emission line observations that M 82 has a family of stellar $x_{2}$-orbits which extends to $\sim \pm 5^{\prime \prime}(\sim \pm 90 \mathrm{pc})$ projected radial distance. The turn-around velocity, which is approximately the line-of-sight velocity of the $x_{2}$-orbits, is $\sim \pm 150 \mathrm{~km} \mathrm{~s}^{-1}$; the projected velocity gradient is $\sim 150 \mathrm{~km} \mathrm{~s}^{-1} / 5^{\prime \prime} \approx$ $30 \mathrm{~km} \mathrm{~s}^{-1}(\operatorname{arcsec})^{-1}=1.7 \mathrm{~km} \mathrm{~s}^{-1} \mathrm{pc}^{-1}$.

\subsection{The cusped orbits ( $\left.5^{\prime \prime} \leq \theta \leq 15^{\prime \prime}\right)$}

As evident from Fig. 3, outside the $x_{2}$-orbits and between $\sim 5^{\prime \prime}(\sim 100 \mathrm{pc})$ and $\sim 15^{\prime \prime}(\sim 250 \mathrm{pc})$ radial distance, the observation does not show stars and ionized gas which follow the predicted $x_{1}$-orbits. In this region (and for this slit position) there occurs on the eastern and western side of the galactic center an apparent transition of stars and gas from a slower rotation on the "outer" $x_{1}$-orbits (Sect. 4.3) to the faster rotation on the $x_{2}$-orbits. This transition gives the impression that the region is primarily occupied, though asymmetrically, by stars and gas moving on cusped orbits.

However, in the cusped orbit region the gas (and perhaps also some stars) "spray" from the trailing $x_{1}$-orbits onto the leading $x_{1}$-orbits, usually producing shocks and dust lanes, as seen in other bar galaxies. The cusped orbit region of M 82 coincides approximately with the dust lanes proposed by Larkin et al. (1994) and Achtermann \& Lacy (1995), and predicted in simulations of gas flow in stellar bars (Athanassoula 1992). Following Larkin et al., and Fig. 2, the western dust lane of M 82 lies mainly in front of the stars of the bar which explains the visual stellar extinction of $A_{\mathrm{V}} \approx 4-6 \mathrm{mag}$ measured in this direction; the eastern dust lane lies mainly behind the stars of the bar which explains the measured lower visual stellar extinction of $A_{\mathrm{V}} \approx 1.5-3 \mathrm{mag}$. However, the observed apparent asymmetry in the $p-v$ pattern of the cusped orbit region is only partially due to extinction in the dust lanes. The reason that in the observation of Fig. 3 mainly a western negative-velocity branch and an eastern positive-velocity branch is seen, is probably due to significant spraying of gas (and some stars) which results in a similar $p-v$ pattern as the incomplete cusped orbits. To clarify the situation, a raster of long-slit observations parallel to the major axis is required.

From the observations published by Wills et al. (2000, their Fig. 9) we notice that the cusped orbit region contains $\mathrm{CO}$ on the western side of the galactic center and primarily on the southern side of the galactic plane. H I and
[NeII] is marginally seen on cusped orbits on the western side of the galactic center and on the northern side of the galactic plane. It is important to note that in the cusped orbit region the velocity of $\mathrm{H}$ I absorption features (seen against continuum sources) however follows the straight branch of the cusped orits and the $x_{1}$-orbits (Wills et al. 2000, their Fig. 10).

The prominent (CO) molecular gas lobes are located between $5^{\prime \prime} \lesssim \theta \lesssim 20^{\prime \prime}(90 \mathrm{pc} \lesssim r \lesssim 350 \mathrm{pc})$ radial distance and thus occupy to a large extent the region of the cusped orbit stars. The effect of spraying may explain the strong accumulation of the molecular gas in this region. The CO gas follows the motion of the "outer" $x_{1}$-orbit stars (Neininger et al. 1998).

The [NeII] observation by Achtermann \& Lacy (1995; Fig. 4) shows a -70 to $-185 \mathrm{~km} \mathrm{~s}^{-1}$ velocity component (called $\left.W_{2}\right)$ between approximately $-7^{\prime \prime}(-120 \mathrm{pc}$ west) and $-13^{\prime \prime}\left(-230 \mathrm{pc}\right.$ west) radial distance. The $W_{2}$ component is also seen in CO and H I (Wills et al. 2000, their Fig. 9) at the western side of the unusual "hole"feature, in the galactic plane and on the southern side. Wills et al. suggest that the eastern side of this "hole"feature is gas moving along the $x_{2}$-orbits and that the western side, which corresponds to $W_{2}$, is produced by gas moving along the cusped orbits. Since an equivalent component has not convincingly been identified on the eastern side of the galaxy, Wills et al. suggest that the bar in M 82 is asymmetric. As an alternative explanation for the asymmetric orbits of the gas, Weiß et al. $(1999,2001)$ interpret the "hole"-feature including the $W_{2}$-component as an expanding super-bubble, containing near its center the SNR 41.9+58, and with molecular gas flowing out into the halo. In the observation of the stellar Ca II line we find no evidence of the $W_{2}$-component and of the western "hole"feature, suggesting that these components are features of the gas only.

In the $2.2 \mu \mathrm{m}$ major axis profile published by Larkin et al. (1994, their Fig. 2) the cusped orbit region corresponds to the well defined and symmetric "inner plateau" (effective radius $\Theta_{\mathrm{c}} \approx 10^{\prime \prime}, R_{\mathrm{c}} \approx 175 \mathrm{pc}$ ) and the sharp transition ( $30 \mathrm{pc}$ wide) to the "outer plateau". The light distribution and the major axis velocity distribution do not indicate an asymmetric bar.

\subsection{The "outer" $x_{1}$-orbits $\left(15^{\prime \prime} \leq \theta \leq 30^{\prime \prime}\right)$}

As evident from Fig. 3, outside the cusped orbit region $\left(15^{\prime \prime} \lesssim \theta\right.$, i.e. $\left.250 \mathrm{pc} \lesssim r\right)$ the stars and gas move on $x_{1}$-orbits which extend to a radial distance of $\sim 30^{\prime \prime}(\sim 500 \mathrm{pc})$. The turn-around velocity of the $x_{1}$-orbit stars at $\sim \pm 30^{\prime \prime}(r \approx 500 \mathrm{pc})$ radial distance is $\sim \pm 125-150 \mathrm{~km} \mathrm{~s}^{-1}$; the projected velocity gradient of the $x_{1}$-orbits is $125 \mathrm{~km} \mathrm{~s}^{-1} / 30^{\prime \prime} \approx 6 \mathrm{~km} \mathrm{~s}^{-1}(\operatorname{arcsec})^{-1}=$ $0.35 \mathrm{~km} \mathrm{~s}^{-1} \mathrm{pc}^{-1}$.

In the $2.2 \mu \mathrm{m}$ major axis profile measured by Larkin et al. (1994, their Fig. 2) the region of the "outer" $x_{1}$-orbits corresponds to the well defined and symmetric "outer plateau" (effective radius $\Theta_{1} \approx 30^{\prime \prime}, R_{1} \approx 500 \mathrm{pc}$ ). 


\subsection{The disk component}

As evident from Fig. 1 and Wills' et al. (2000) observations, projected onto the center region of M 82, but in particular outside the bar, gas and stars are observed which belong to the galactic disk. Projected onto the center region, this material is seen in $21 \mathrm{~cm}-\mathrm{H}_{\mathrm{I}}$ (absorption) with a projected velocity gradient of $\sim 3 \mathrm{~km} \mathrm{~s}^{-1}(\operatorname{arcsec})^{-1} \approx$ $0.2 \mathrm{~km} \mathrm{~s}^{-1} \mathrm{pc}^{-1}$ (Wills et al. 2000, their Figs. 9 and 11). This foreground disk material is also seen with the same velocity gradient in blue wavelength emission lines and the blue stellar CaIIK line at $3933 \AA$ (McKeith et al. 1993, their Fig. 3). At blue wavelengths the average extinction towards the center is so high that only the slowly moving material of the outer galactic disk is seen. Outside the bar $(500 \mathrm{pc} \lesssim r)$, the CaII absorption line of the disk stars indicates a projected velocity gradient of $\sim 1 \mathrm{~km} \mathrm{~s}^{-1}(\operatorname{arcsec})^{-1}=0.1 \mathrm{~km} \mathrm{~s}^{-1} \mathrm{pc}^{-1}$ (Fig. 1).

\section{The mass of the stellar bar}

In the derivation of masses $M(r) \approx r v^{2} / G$ [with $G$ the gravitation constant] we assume that the turn-around velocity $v\left(r_{x 2}\right)$ of the $x_{2}$-orbit material determines the mass $M\left(x_{2}\right)$ of the $x_{2}$-orbit stars (inside $\theta_{x 2} \approx 5^{\prime \prime}$, i.e. $\left.r_{x 2} \approx 90 \mathrm{pc}\right)$, and that the turn-around velocity $v\left(r_{x 1}\right)$ of the "outer" $x_{1}$-orbit material determines the mass $M\left(x_{1}\right)$ of the stars and gas concentrated along the bar (inside $\Theta_{1}=\theta_{x 1} \approx 30^{\prime \prime}$, i.e. $R_{1}=r_{x 1} \approx 500 \mathrm{pc}$ ). The corresponding masses are given in Table 2 .

We find that the mass of the $x_{2}$-orbit stars is $\sim 15 \%$ of the mass of the $x_{1}$-orbit stars (including the cusped orbit stars), and that the mass of the bar is $M\left(x_{1}\right)+M\left(x_{2}\right)=$ $M_{\text {bar }} \approx 2 \times 10^{9} M_{\odot}$. When adopting for the mass of M 82 inside the radius of $\sim 2 \mathrm{kpc}$ the value $M_{\mathrm{M} 82}(<2) \approx$ $1 \times 10^{10} M_{\odot}$ determined by Sofue et al. (1992), we find that the mass concentrated in the bar is $\sim 20 \%$ of the galaxy mass. However, if we use the mass distribution of M 82 derived by Götz et al. (1990; see also Förster Schreiber $2001)$, the mass inside the radius of $r \lesssim 2.7 \mathrm{kpc}$ is $M(<2.7) \approx 4 \times 10^{9} M_{\odot}$ and thus only half of the mass given by Sofue. When using the mass of M 82 determined by Götz et al., the bar contains $\sim 40 \%$ of the galaxy mass. The mass of the gas and dust $M(\mathrm{~g}+\mathrm{d})$ in the region of the bar is $\sim 40 \%$ of the stellar mass of the bar.

We get similar mass ratios from an analysis of the $2.2 \mu \mathrm{m}$ major axis profile measured by Larkin et al. (1994). If the $2.2 \mu \mathrm{m}$ radiation is primarily due to (late-type) stars, and if the observed brightness of the radiation is proportional to the number of stars, hence the mass along the line-of-sight, the proportionality of the individual stellar mass components is

$M\left(x_{i}\right) \propto 10^{0.4 \Delta m(\mathrm{o}, i)} \times R_{i} \times M_{\mathrm{o}}$

with $i=1,2, c$ for the $x_{1}$-orbit stars, $x_{2}$-orbit stars, and cusped orbit stars. $\Delta m(\mathrm{o}, i)=I_{\mathrm{o}}-I_{i}$ is the magnitude difference between the stellar component $\left(I_{i}\right)$ and the stellar background $\left(I_{\mathrm{o}}\right)$, measured just outside the bar.
$R_{i}$ is the radial extent of the stellar component; $M_{\mathrm{O}}$ is a scaling factor. When using the parameters of Table 3 we obtain $M\left(x_{2}\right) \approx 25 \times M_{\mathrm{o}}, M\left(x_{1}\right) \approx 75 \times M_{\mathrm{o}}$, and $M\left(x_{\mathrm{c}}\right) \approx 90 \times M_{\mathrm{o}}$. We find again that $\sim 15 \%$ of the mass is concentrated in $x_{2}$-orbit stars, $\sim 50 \%$ of the mass in $x_{1}$-orbit stars, and $\sim 30 \%$ of the mass in cusped orbit stars.

We assume in these estimates that the dominant amount of stellar mass is concentrated in the late-type stars of the bar, and not in young massive stars produced in the starburst. When following McLeod et al. (1993), the mass of the stars formed in the starburst is $M_{\mathrm{stb} *} \approx 3 \times 10^{8} M_{\odot}$, which is approximately $1 / 10$ of the mass of the bar. The newly formed stars are primarily located near the molecular ring so that the $x_{2}$-orbit region contains only a negligible percentage of young massive stars. The mass $M\left(x_{2}\right)$ (Table 2 ) is therefore primarily due to the late-type stars of the bar.

\section{Summary}

This paper shows the stellar rotation curve of M 82 up to $\pm 1700 \mathrm{pc}$ radial distance, fully traced from the near-IR CaII photospheric absorption line of late-type stars. The position-velocity analysis of this line gives direct evidence of the stellar bar in M82, in particular since the observation shows the $x_{2}$-orbit stars at the center of the galaxy, hidden behind substantial visual extinction but visible at (near-)IR wavelengths. The observed $x_{1}$ and $x_{2}$-orbit structure agrees with the stellar light distribution (at $2.2 \mu \mathrm{m}$ ) of the bar and with gas moving on the stellar orbits, although local disturbances of the gas motion do occur. The $x_{2}$-orbits extend to $r \lesssim 100 \mathrm{pc}$; the $x_{1}$-orbits extend to $r \approx 500 \mathrm{pc}$; the cusped orbits are located between $100 \mathrm{pc} \lesssim r \lesssim 250 \mathrm{pc}$. The cusped orbits are not clearly visible in our observation probably because in this region there exists significant spraying of gas, and probably also of some stars. The orbital revolution time of the $x_{2}$-orbit stars is $\sim 2 \mathrm{Myr}(r \approx 100 \mathrm{pc}$, $v \approx 150 \mathrm{~km} \mathrm{~s}^{-1}$ ) and of the $x_{1}$-orbit stars $\sim 10-15 \mathrm{Myr}$ $\left(r \approx 500 \mathrm{pc}, v \approx 125 \mathrm{~km} \mathrm{~s}^{-1}\right)$.

The mass of the bar is $\sim 2.0 \times 10^{9} M_{\odot}$. The $x_{2}$-orbit stars contain $\sim 15 \%$ of the mass of the bar. Dependent of the adopted mass model, i.e. the one determined by Sofue et al. (1992) or Götz et al. (1990), the stellar bar contains approximately $20-40 \%$ of M 82 's mass. This mass has a dominating influence on the kinematics of the stars and the gas, at least in the $\sim 1 \mathrm{kpc}$ center region. The stellar bar (at $2.2 \mu \mathrm{m}$ ) defines the kinematic center of M 82 rather than the center region gas, which constitutes only $\sim 40 \%$ of the stellar mass.

Figure 5 shows, in an idealized way, the location of the stellar bar superimposed on an $\mathrm{HST}_{\alpha}$ image. The bar (long side of the box) is roughly aligned to the galactic plane of M 82; the stars move roughly in this plane. The thickness of the bar, in this image, is taken to extent $\sim \pm 10^{\prime \prime}(\approx 150 \mathrm{pc}$ ) above the galactic plane. This value is taken from the K-image published by Telesco et al. (1991); the image shows late-type stars which are the major 
Table 2. Mass of M 82's stellar bar (inside $r \lesssim 500 \mathrm{pc}$ ).

\begin{tabular}{|c|c|c|c|c|c|}
\hline Region & $\begin{array}{l}\text { Radius } \\
\quad \theta-r\end{array}$ & $\begin{array}{l}\text { Velocity } v \text { at } r \\
\quad\left(\mathrm{~km} \mathrm{~s}^{-1}\right)\end{array}$ & $\begin{array}{l}\text { Velocity Gradient } \\
\left(\mathrm{km} \mathrm{s}^{-1} /{ }^{\prime \prime}\right)\end{array}$ & $\begin{array}{l}\text { Mass } \\
\left(M_{\odot}\right)\end{array}$ & Ref. \\
\hline $\begin{array}{c}\text { Stellar Bar Components } \\
x_{2}-\text { region } \\
x_{1}-\text { region }^{\mathrm{a})} \\
\text { Total }\left(\boldsymbol{x}_{1}+\boldsymbol{x}_{2} \text { region }\right)\end{array}$ & $\begin{array}{c}5^{\prime \prime}-90 \mathrm{pc} \\
30^{\prime \prime}-500 \mathrm{pc}\end{array}$ & $\begin{array}{l}150 \\
125\end{array}$ & $\begin{array}{c}30 \\
6\end{array}$ & $\begin{array}{c}M\left(x_{2}\right)=2.9 \times 10^{8} \\
M\left(x_{1}\right)=1.75 \times 10^{9} \\
M\left(x_{1}+x_{2}\right)=2.0 \times 10^{9}\end{array}$ & \\
\hline $\begin{array}{c}\text { Mass Model (Götz et al.) } \\
\text { Mass Model (Förster-S. et al.). }\end{array}$ & $\begin{array}{l}30^{\prime \prime}-500 \mathrm{pc} \\
30^{\prime \prime}-500 \mathrm{pc}\end{array}$ & & & $\begin{array}{l}M=1.9 \times 10^{9} \\
M=1.6 \times 10^{9}\end{array}$ & $\begin{array}{l}1 \\
2\end{array}$ \\
\hline Molecular Gas \& Dust & $\begin{array}{l}\sim 30^{\prime \prime}-500 \mathrm{pc} \\
\sim 40^{\prime \prime}-700 \mathrm{pc} \\
\sim 40^{\prime \prime}-700 \mathrm{pc}\end{array}$ & & & $\begin{array}{c}M\left(\mathrm{H}_{2}\right)=2.3 \times 10^{8} \\
M(\mathrm{~g}+\mathrm{d})=8 \times 10^{8} \\
M(\mathrm{~d})=8 \times 10^{6}\end{array}$ & $\begin{array}{c}3 \\
4 \\
3,4\end{array}$ \\
\hline Young starburst stars & $\sim 30^{\prime \prime}-500 \mathrm{pc}$ & & & $M_{\mathrm{stb} *} \approx 3 \times 10^{8}$ & 5 \\
\hline Galaxy Mass & $\begin{array}{l}\sim 120^{\prime \prime}-2 \mathrm{kpc} \\
\sim 150^{\prime \prime}-2.7 \mathrm{kpc}\end{array}$ & & & $\begin{array}{c}M_{\mathrm{M} 82} \approx 1 \times 10^{10} \\
M_{\mathrm{M} 82} \approx 4 \times 10^{9}\end{array}$ & $\begin{array}{l}6 \\
1\end{array}$ \\
\hline
\end{tabular}

a) Including the cusped orbit stars.

1) From Götz et al. (1990) mass model.

2) From Förster Schreiber et al. (2001) mass model.

3) Weiß et al. (2001): $\mathrm{H}_{2}$ mass.

4) $230 \mathrm{GHz}$ continuum observations (Krügel et al. 1990) and Thuma et al. (2000): gas and dust mass $\left(M_{\mathrm{d}} \approx M_{\mathrm{g}} / 100\right)$.

5) McLeod et al. (1993)

6) Sofue et al. (1992).

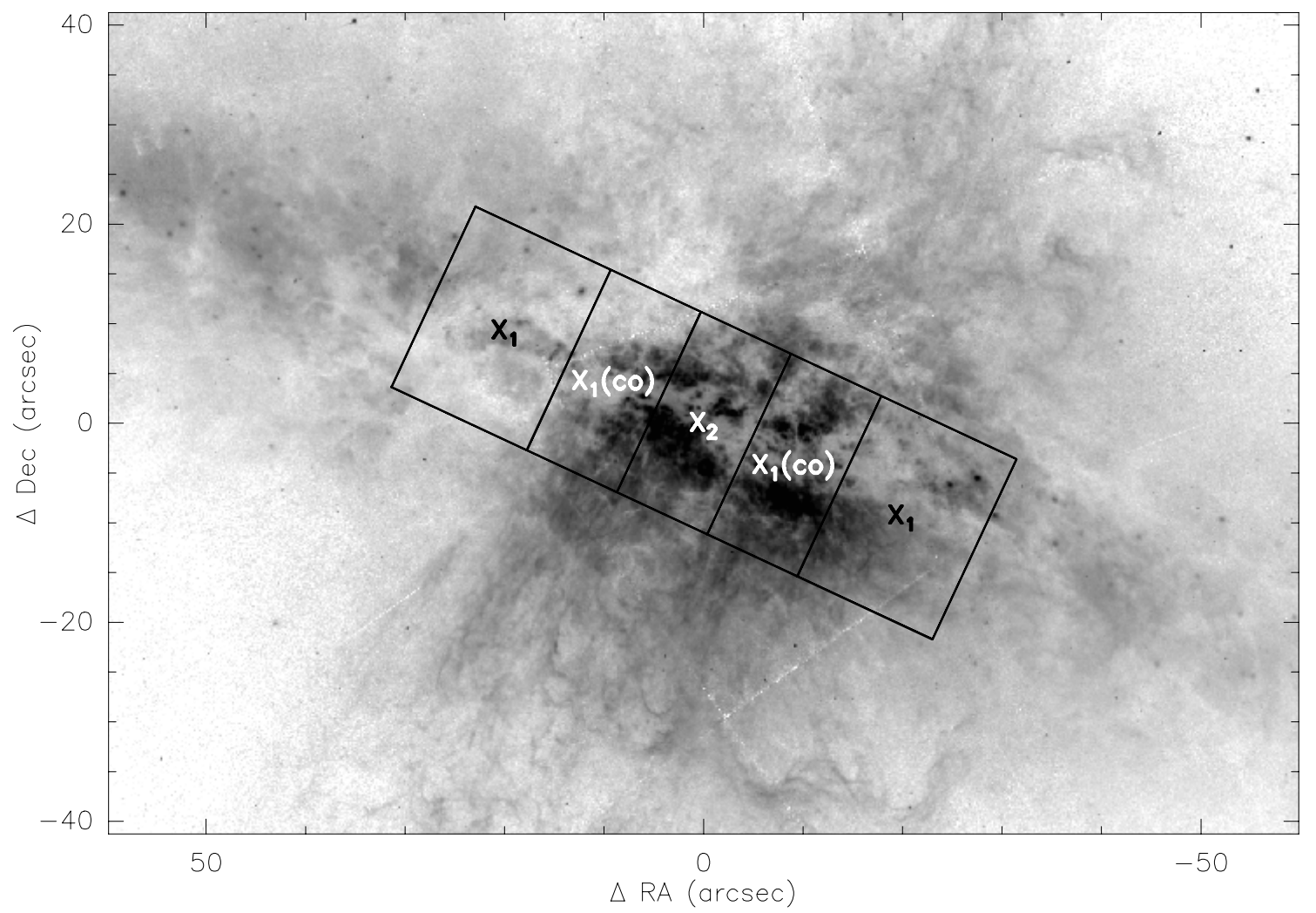

Fig. 5. $\mathrm{H}_{\alpha}$ image of M 82 taken with the HST. The box shows an idealized stellar bar, aligned roughly along the major axis. The region of the $x_{2}$-orbit stars, the cusped orbit stars $\left(x_{1}(\mathrm{co})\right)$, and the "outer" $x_{1}$-orbit stars is indicated (see Fig. 3). The thickness of the bar is $\sim \pm 10^{\prime \prime}(\sim \pm 150 \mathrm{pc})$ as evident from the K-image taken by Telesco et al. (1991). For more detail and designation of the objects see the image published by O'Connell et al. (1995). East is to the left, North at the top. 
Table 3. Stellar brightness of the bar (along major axis).

\begin{tabular}{lcccc}
\hline Stellar Component & $2.2 \mu \mathrm{m}$ Profile $^{\mathrm{a})}$ & $\begin{array}{c}\text { Radius }^{\mathrm{b})} \\
\Theta_{i}-R_{i}\end{array}$ & $\begin{array}{c}\text { Brightness }^{\mathrm{a})} \\
I_{i}\left[\mathrm{mag}\left({ }^{\prime \prime}\right)^{-2}\right]\end{array}$ & $10^{0.4 \Delta m(\mathrm{o}, i)}$ \\
\hline Background (o) & - & 14.5 & 1 \\
$x_{1}$ stars (1) & Outer Plateau & $30^{\prime \prime}-500 \mathrm{pc}$ & 13.5 & 2.5 \\
Cusped orbit stars (c) & Inner Plateau & $10^{\prime \prime}-175 \mathrm{pc}$ & 12.1 & 8.7 \\
$x_{2}$ stars (2) & {$[$ Peak $]$} & $2^{\prime \prime}-35 \mathrm{pc}^{\mathrm{c})}$ & 11.7 & 12.6 \\
\hline
\end{tabular}

a) From Larkin et al. (1994), their Fig. 2.

b) See Sect. 4 . The full extent of the component is $2 \times R_{i}$.

c) Weighted by the exponential spatial light distribution.

constituents of the bar. Figure 5 shows the region of the $x_{2}$-orbit stars $\left(|\theta| \lesssim 5^{\prime \prime}\right)$, the region to which the cusped orbit stars extent $\left(x_{1}(\mathrm{co}): 5^{\prime \prime} \lesssim|\theta| \lesssim 15^{\prime \prime}\right)$, and the full extent of the $x_{1}$-orbit stars $\left(|\theta| \lesssim 30^{\prime \prime}\right)$. Because of the large visual extinction, the $\mathrm{H}_{\alpha}$ image shows mainly the pheripheral parts of M 82 and the outflow along the minor axis, especially on the SE side (Shopbell \& Bland-Hawthorn 1998). The image shows heavy extinction on the NE side in the region of the $x_{1}$-orbits. This is foreground extinction and not the dust lane assumed to be produced by sprays (see Fig. 2); at this side of the center (NE) the dust lane lies on the far side of the galaxy. The minor axis outflow, which breaks out of the galactic disk at a height of $\sim \pm 150 \mathrm{pc}$ (McKeith et al. 1995), is confined to the inner region of the bar, i.e. approximately the region of the cusped orbits where the molecular ring is located. This is the main area of the starburst.

Acknowledgements. We appreciated the discussions with D. Downes (IRAM) on this subject. We thank the referee D. Elmegreen for her comments. Figure 5 is based on observations made with the NASA/ESA Hubble Space Telescope, obtained from the data archive at the Space Telescope Science Institute. The STScI is operated by the Association of Universities for Research in Astronomy, Inc., under NASA contract NAS 5-26555.

\section{References}

Achtermann, J. M., \& Lacy, J. H. 1995, ApJ, 439, 163

Athanassoula, E. 1992, MNRAS, 259, 345

Beck, S. C., Lacy, J. H., Baas, F., \& Townes, C. H. 1978, ApJ, 226,545

Binney, J., Gerhard, O. E., Stark, A. A., Bally, J., \& Uchida, K. I. 1991, MNRAS, 252, 210

Chesterman, J., \& Pallister, W. 1980, MNRAS, 191, 349

Contopoulos, G., \& Mertzanides, C. 1977, A\&A, 61, 477

Downes, D., Reynaud, D., Solomon, P. M., \& Radford, S. J. E. 1996. ApJ, 461, 186

Förster Schreiber, N. M., Genzel, R., Lutz, D., \& Kunze, D. 2001, ApJ, 552, 544
Freedman, W., Hugles, S. M., Madore, B. F., et al. 1994, ApJ, 427, 628

Götz, M., McKeith, C. D., Downes, D., \& Greve, A. 1990, A\&A, 240, 52

Jaschek, C., \& Jaschek, M. J. 1995, The Behavior of Chemical Elements in Stars (Cambridge University Press, UK)

Krügel, E., Chini, R., Klein, U., Wielebinski, R., \& Zylka, R. 1990, A\&A, 240, 232

Larkin, J. E., Graham, J. R., Matthews, K., et al. 1994, ApJ, 420, 159

McKeith, C. D., Castles, J., Greve, A., \& Downes, D. 1993, A\&A, 272, 98

McKeith, C. D., Greve, A., Downes, D., \& Prada, F. 1995, A\&A, 293, 703

McLeod, K. K., Rieke, G. H., Rieke, M. J., \& Kelly, D. M. 1993, ApJ, 412, 111

Neininger, N., Guélin, M., Klein, U., García-Burillo, S., \& Wielebinski, R. 1998, A\&A, 339, 737

Notni, P., \& Bronkalla, W. 1983, in Internal Kinematics and Dynamics of Galaxies, IAU Symp. 100, ed. E. Athanassoula (Reidel Dordrecht), 67

O'Connell, R. W., Gallagher, J. S., Hunter, D. A., \& Colley, W. N. 1995, ApJ, 446, L1

Saito, M., Sasaki, M., Kaneko, N., Nishimura, M., \& Toyama, K. 1984, PASJ, 36, 305

Sakai, S., \& Madore, B. F. 1999, ApJ, 526, 599

Seaquist, E. R., Carlstrom, J. E., Brynat, P. M., \& Bell, M. B. 1996, ApJ, 465, 691

Shen, J., \& Lo, K. Y. 1995, ApJ, 445, L99

Shopbell, P. L., \& Bland-Hawthorn 1998, ApJ, 493, 129

Sofue, Y., Reuter, H.-P., Krause, M., Wielebinski, R., \& Nakai, N. 1992, ApJ, 395, 126

Telesco, C. M., Campins, H., Joy, M., Dietz, K., \& Decher, R. 1991, ApJ, 369, 135

Thuma, G., Neininger, N., Klein, U., \& Wielebinski, R. 2000, A\&A, 358, 65

Visvanathan, N., \& Sandage, A. 1972, ApJ, 176, 57

Weiß, A., Walter, F., Neininger, N., \& Klein, U. 1999, A\&A, 345, L23

Weiß, A., Neininger, N., Hüttemeister, S., \& Klein, U. 2001, A\&A, 365, 571

Wills, K. A., Das, M., Pedlar, A., Muxlow, T. W. B., \& Robinson, T. G. 2000, MNRAS, 316, 33 